\title{
UNSTEADY DYNAMICS OF FREE-INTERFACE GRAVITATIONAL LIQUID SHEET FLOWS
}

\author{
Alessandro Della Pia ${ }^{1}$, Matteo Chiatto ${ }^{2}$ and Luigi de Luca ${ }^{3}$ \\ 1,2,3 Department of Industrial Engineering, Università degli Studi di Napoli “Federico II”, Naples, Italy \\ 1 alessandro.dellapia@unina.it \\ 2 matteo.chiatto@unina.it \\ 3 deluca@unina.it
}

Key words: Interfacial flows, Computational Fluid Dynamics, Instabilities

\begin{abstract}
Numerical simulations of gravitational planar liquid sheet flows, interacting with unconfined gaseous environments located on both sides of the liquid phase, are performed through Volume-of-Fluid (VOF) technique. The global unsteady dynamics of the non-parallel flow is analyzed by perturbing the initial steady configuration by means of a Gaussian bump in the transverse velocity component of relatively very small amplitude, thereby exciting sinuous modes. Thanks to the development of a theoretical linear one-dimensional model, more physical insights are gained on the flow system. It is found that surface tension plays a stabilizing role for the gravitational sheet, and for relatively high values of density ratio $r_{\rho}$ of gaseous-to-liquid phases it becomes unstable. An analogy is shown between the global unstable behavior exhibited by the liquid sheet as $r_{\rho}$ increases, and the shear-induced global instability found by Tammisola et al. ["Surface tension-induced global instability of planar jets and wakes", J. Fluid Mech. 713, 632-658 (2012)] for planar jet and wake flows of two immiscible fluids in the presence of surface tension.
\end{abstract}

\section{INTRODUCTION}

Liquid sheet flows issuing into a gaseous environment have being studied since the middle of the last century [1], due to their wide scientific and industrial interest, which involves fuel atomization, coating deposition, paper making, and other applications. The literature includes a wide variety of geometrical and physical configurations, which span both planar [2] and round [3] geometries, both high [4] and low speed (gravitational) [5] flows, both quiescent [6] and co-flowing air [7] ambients. Notwithstanding the large amount of research efforts, some aspects of the unsteady dynamics of liquid sheet flows are the object of ongoing studies and some are still open. As an example, the analysis of the singularity exhibited by the equation governing the unsteady subcritical dynamics of the so called liquid nappe, namely the freely falling liquid sheet interacting with a one-sided closed air chamber, has been fully accomplished only a few years ago [8], while the extension of the theoretical modeling of the frequency jump to liquid sheets flowing in unconfined environments is the object of ongoing research.

The present paper deals with the two-dimensional unsteady response to impulse forcing of planar thin liquid sheets, interacting with unconfined gaseous environments located on both sides of the liquid phase. 
The liquid velocity is relatively low so that the relevant Froude number is small and the gravitation effects have to be taken into account. As a consequence, the base flow is not parallel, i.e. it is not invariant with respect to translations along the motion direction. The goal of the investigation is the determination of the unsteady response (i.e. eigenmodes and eigenvalues) of the flow system in supercritical regimes (inlet Weber number We greater than unity), which is obtained through direct numerical simulation of the steady field forced by an impulse disturbance applied at a specified vertical station. The numerical simulation is performed by means of the Volume-Of-Fluid (VOF) technique, which has been recently employed to explore the stability of forced planar liquid sheets (jets) at relatively high Weber (and Froude) numbers by Schmidt and Oberleithner [9]. The analysis of the global behavior of the sheet is completed by the computation of its natural frequencies, and, furthermore, eigenmodes and eigenvalues will be compared with predictions made by means of a simplified one-dimensional modeling, making use of a linear stability approach. Finally, two-dimensional patterns of unstable liquid sheets are presented and discussed, providing an analogy with the global behavior of planar sheets (jets and wakes) studied by Tammisola et al. [10].

\section{VOF NUMMERICAL SIMULATION}

The mathematical modeling of the interface separating immiscible fluids is a crucial aspect to obtain accurate numerical predictions of multiphase flows behavior. A current approach consists in deriving the governing equations for two-phase flows following the single-phase approach [11]. Given the assumption of two-dimensional incompressible flow, continuity and momentum equations in the so called one-fluid

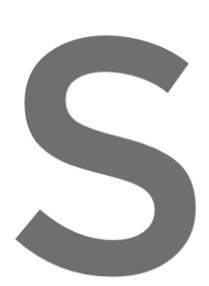
formulation read as
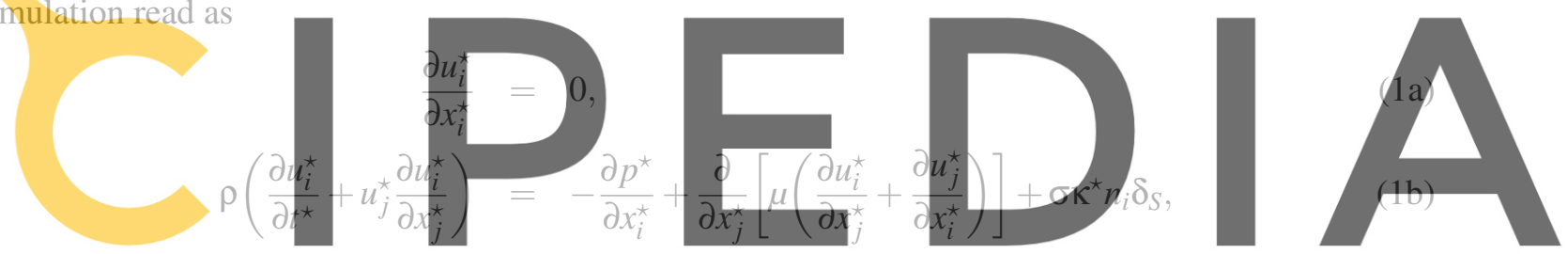

with $\left(u_{1}^{\star}, u_{2}^{\star}\right)^{T}=\left(u^{\star}, v^{\star}\right)^{T}$ representing the velocity vector, $p^{\star}$ the pressure, $\left(x_{1}^{\star}, x_{2}^{\star}\right)^{T}=\left(x^{\star}, y^{\star}\right)^{T}$ the spa-

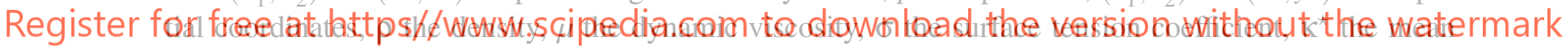
interface curvature and $\left(n_{1}, n_{2}\right)^{T}=\left(n_{x}, n_{y}\right)^{T}$ the outward pointing normal vector. Note that all the dimensional quantities, except the fluid material properties $(p, \mu, \sigma)$, are denoted with the superscript $*$. The phase boundary is the only region of the domain where the term $\sigma \kappa^{\star} n_{i} \delta_{S}$, accounting for the effect of the surface tension, is different from zero. Indeed $\delta_{S}$ is a Dirac distribution function, equal to 1 at the interface, 0 otherwise. Therefore, density and viscosity fields are evaluated as

$$
\begin{aligned}
& \rho=\rho_{a}+\left(\rho_{l}-\rho_{a}\right) C, \\
& \mu=\mu_{a}+\left(\mu_{l}-\mu_{a}\right) C,
\end{aligned}
$$

where subscripts $a$ and $l$ refer to gaseous ( $a$ stands for ambient) and liquid phases, respectively, and $C\left(x^{\star}, y^{\star}, t^{\star}\right)$ is the volume fraction, a (dimensionless) Heaviside function equal to the liquid-to-total volume ratio. Computational cells where $C=1$ are located in the liquid phase and cells with $C=0$ are situated in the gaseous phase; $0<C<1$ for cells crossing the interface. An additional equation is therefore required to localize the interface, namely the volume fraction advection equation

$$
\frac{\partial C}{\partial t^{\star}}+\frac{\partial C u_{i}^{\star}}{\partial x_{i}^{\star}}=0,
$$




\begin{tabular}{|lr|}
\hline$\left(0 ; L^{\star} / 2\right)$ & $\left(L^{\star} ; L^{\star} / 2\right)$ \\
$y^{\star}$ & \\
$\left(0 ; H_{i}^{\star} / 2\right)$ & $\rho_{a}, \mu_{a}$ \\
\hline & $\rho_{l}, \mu_{l}$ \\
\hline$x^{\star}$ & \\
$\left(0 ;-H_{i}^{\star} / 2\right)$ & \\
$\left(0 ;-L^{\star} / 2\right)$ & $\left(L^{\star} ;-L^{\star} / 2\right)$ \\
\hline
\end{tabular}

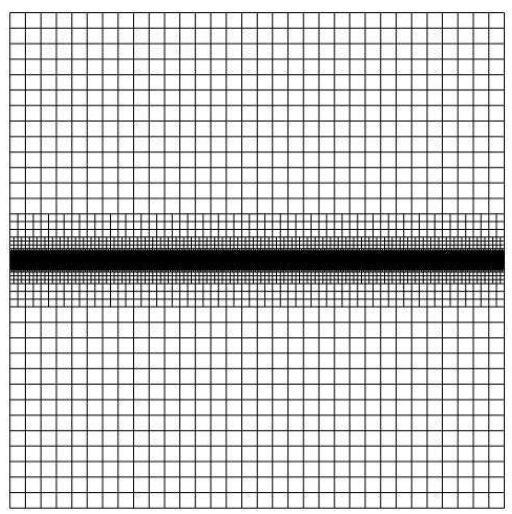

Figure 1: Schematic of computational domain (left) and of adaptive grid (right). The sheet of length $L^{\star}$ falls under gravity along $x^{\star}$ direction. Red lines define the initial interface shape, which is magnified by a factor of 10 along $y^{\star}$ direction in left panel.

which, coupled with the system of Eqs. (1a - 1b), closes the problem. The equations discussed above are solved using the finite volume method in the open-source code BASILISK (http://basilisk. fr); for a detailed description of the implemented numerical schemes the reader can refer to Popinet $[12,13]$.

\subsection{Computational layout}

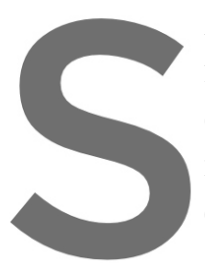

A schematic descriptio

for computational convent

domain is a square whose

inlet sheet thickness lodatecto

coincides with the mean pr

shape of the interface,
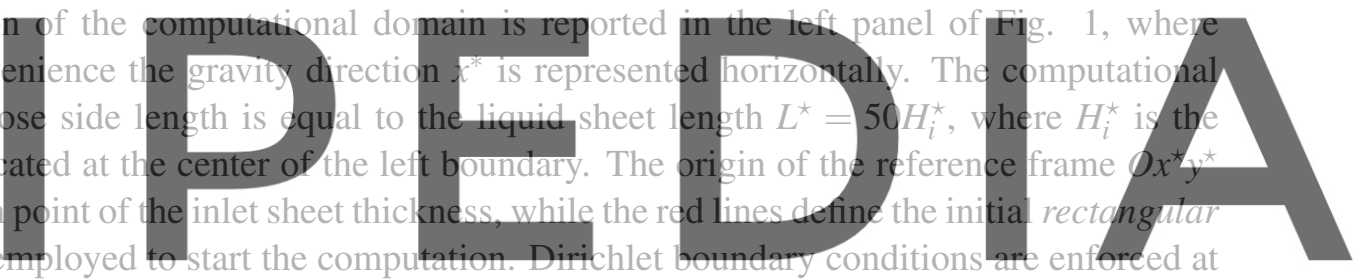

the inlet: in the liquid region, around the symmetry axis, a fully developed parabolic velocity profile is

Register fönpfree, aitdhitteshdivibvisv:scipedia.com to download the version without the watermark

$$
u^{\star}=\frac{3}{2} U_{i}^{\star}\left[1-\left(\frac{2 y^{\star}}{H_{i}^{\star}}\right)^{2}\right], \quad v^{\star}=0, \quad C=1,
$$

where $U_{i}^{\star}$ represents the mean liquid velocity at the inlet. On the remaining part of the left edge (gaseous phase) a no-slip condition is imposed. The top and lower edges are equipped with Neumann boundary conditions for all variables. At the right edge a standard outflow condition is considered. The liquid sheet region is initialized with a rectangular interface shape, and the same Poiseuille velocity profile adopted as inlet boundary condition is used throughout the entire sheet length.

The physical quantities involved in the problem can be arranged in 6 dimensionless quantities, following the Buckingham $\pi$ theorem: a possible choice is reported in Table 1. Furthermore, assuming that the liquid phase is water, the liquid density and viscosity, $\rho_{l}$ and $\mu_{l}$, as well as, to within a certain approximation, the surface tension coefficient $\sigma$, are fixed. Note that the surface tension is a chemical property, and thus its value can be assumed independent of the gas-liquid pair of phases. If also the gas viscosity $\mu_{a}$ is considered constant, the independent dimensionless parameters reduce to: 
A. Della Pia, M. Chiatto and L. de Luca

Table 1: Dimensionless parameters corresponding to the Base Case.

\begin{tabular}{ccc}
\hline Name & Relation & Value \\
\hline Gas-to-liquid density ratio & $r_{\rho}=\rho_{a} / \rho_{l}$ & 0.01 \\
Gas-to-liquid viscosity ratio & $r_{\mu}=\mu_{a} / \mu_{l}$ & 0.02 \\
Sheet slenderness ratio & $\varepsilon=H_{i}^{\star} / L^{\star}$ & 0.02 \\
Reynolds number & $\operatorname{Re}=\rho_{l} U_{i}^{\star} H_{i}^{\star} /\left(2 \mu_{l}\right)$ & 420 \\
Froude number & $F r=U_{i}^{\star 2} /\left(g L^{\star}\right)$ & 0.33 \\
Weber number & $W e=\rho_{l} U_{i}^{\star 2} H_{i}^{\star} /(2 \sigma)$ & 2.5 \\
\hline
\end{tabular}

- $W e=\frac{\rho_{l} U_{i}^{\star^{2}} H_{i}^{\star}}{2 \sigma}, F r=\frac{U_{i}^{\star^{2}}}{g L^{\star}}, \varepsilon=\frac{H_{i}^{\star}}{L^{\star}}, r_{\rho}=\frac{\rho_{a}}{\rho_{l}}$,

where $\varepsilon=H_{i}^{\star} / L^{\star}$ is the slenderness parameter, which is intrinsically a small quantity whose order generally ranges from $O\left(10^{-3}\right)$ to $O\left(10^{-2}\right)$. It has been assumed $\varepsilon=0.02$ for all the computations made in the present paper. On the other hand, for the standard case of $g=9.81 \mathrm{~m} / \mathrm{s}^{2}$, the corresponding dimensional parameters are:

\section{- $U_{i}^{\star}, H_{i}^{\star}, L^{\star}, \rho_{a}$.}

It is clearly evident that there exists a 1:1 correspondence between the set of governing dimensionless numbers and the set of physical parameters. This correspondence will be considered in the subsequent parametric investigation, which will be carried out by hypothesizing the variation of one physical parameter at a time (for instance parameters.

Accordingly, the physical quantities (subscript $r$ ):
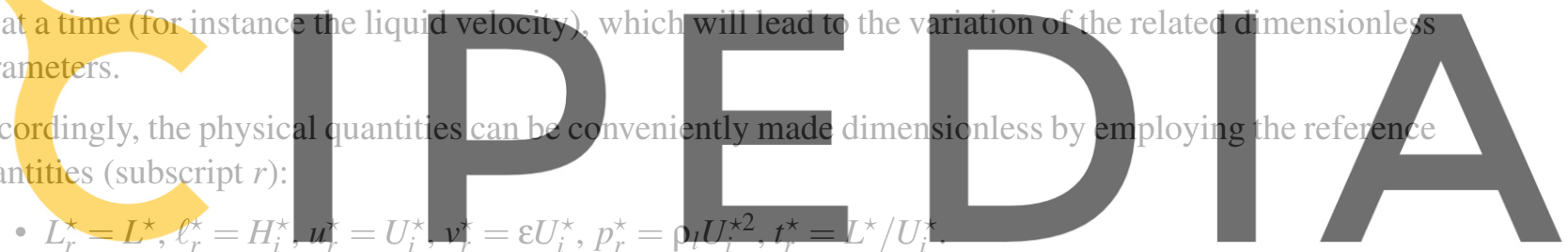

Note that hereafter dimensionless quantities will be denoted without apex $\star$. This Section is concluded

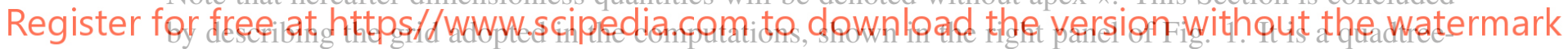

structured grid which retains its maximum level of refinement in a rectangular region containing the entire liquid sheet: $-0.5<y^{\star} / H_{i}^{\star}<0.5,0<x^{\star} / L^{\star}<1$. The maximum resolution is limited to $N=11$ levels of refinement, corresponding to a minimum dimensionless cell edge length of $\Delta x^{\star} / H_{i}^{\star}=0.0244$ (40 grid cells within $H_{i}^{\star}$ ). A detailed description of the adaptive grid refinement strategy is given in [14].

\subsection{Computation of global eigenmodes}

The computation via VOF of the fluid system unsteady response is performed forcing at the initial instant the steady configuration by means of an impulse disturbance applied at a specified $x$ station. The adopted perturbation is a Gaussian bump in the transverse velocity component

$$
v_{f}=A e^{-\frac{1}{2}\left(\frac{x-x_{0}}{\Delta}\right)^{2}},
$$

where $A$ is the amplitude, $x_{0}$ the peak station, and $\Delta^{2}$ represents the variance of the Gaussian function. To guarantee a linear response, at least at the initial instants after the forcing, the following typical parameters have been adopted for all the computations: 
- $A=5 \varepsilon, \Delta=0.1 \varepsilon, x_{0}=0.3$.

As will be seen later in this Section, to validate the simulated results the following decomposition of the unsteady field has been considered:

$$
\phi(t, x, y)=\Phi(x, y)+\phi^{\prime}(t, x, y)
$$

where $\phi$ denotes any physical quantity, $\Phi$ its value at steady conditions (i.e. before the introduction of the Gaussian impulse), and $\phi^{\prime}$ the unsteady fluctuation. The detailed procedure to achieve the steady flow field starting from the initial condition described earlier in this Section is reported in [15], together with the grid convergence properties of the solution.

\subsection{One-dimensional data reduction}

A one-dimensional reduction of VOF data is required to compare the numerically computed global properties of liquid sheet dynamics with the predictions of a linear one-dimensional model that will be introduced in Section 3. This reduction is based on the relationship

$$
\phi_{1 D}=\frac{\sum_{i=1}^{N_{y}} C_{i} \phi_{i}}{\sum_{i=1}^{N_{y}} C_{i}},
$$

where $\phi_{1 D}$ is the spatial-averaged value over the lateral $y$ direction, evaluated at every $x$ station, of the generic physical quantity $\phi ; N_{y}$ is the total number of grid cells at the selected $x$ station, while $C_{i}$ is the volume fraction in the cell $i$. In particular, for $\phi=y$, the average deflection of the sheet, namely the

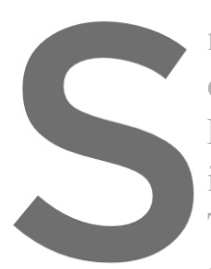
meanline $\ell$, is obtained. of $\phi$ for the liquid phase on Eq. (7) allows for the on importance for the compart Therefore, following t perturbation field $\phi_{1 D}^{\prime}$ has been evaluated as
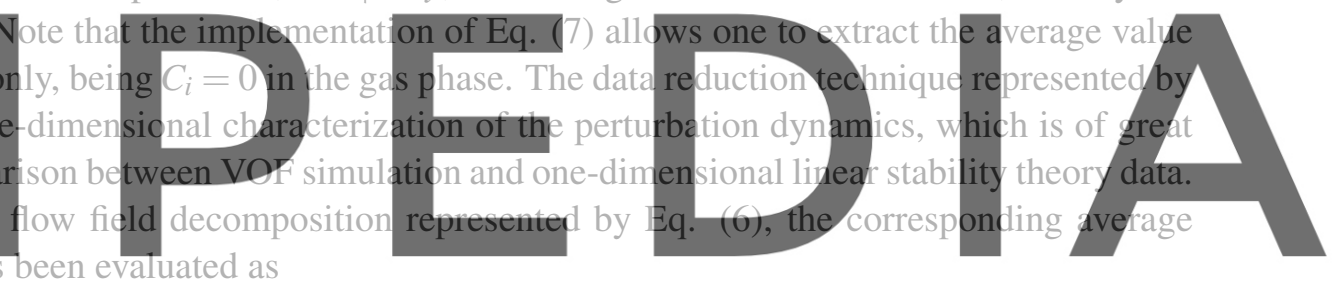

Register for free at https//www.scipedia.c@m $₫ \notin 1$ dow where $\Phi_{1 D}$ is the generic 1D-reduced flow variable at steady conditions.

\section{THEORETICAL ONE-DIMENSIONAL MODELING}

Under the assumption of small perturbations with respect to the base flow and inviscid conditions, a set of two equations governing the perturbations dynamics can be derived starting from the two-dimensional Euler equations; a detailed description of the overall procedure is given in [15]. In the present paper, we only report a physical interpretation of the liquid sheet unsteady dynamics based on a single second order integro-differential equation, which has been derived in [15]:

$$
\frac{\partial^{2} \ell}{\partial t^{2}}-\frac{2}{\pi} \frac{1}{\varepsilon} \frac{\rho_{a}}{\rho_{l}} \frac{1}{H} \int_{0}^{1} \frac{\partial^{2} \ell}{\partial t^{2}} \ln |x-\xi| d \xi+2 U \frac{\partial^{2} \ell}{\partial x \partial t}+U\left(U-\frac{1}{W e}\right) \frac{\partial^{2} \ell}{\partial x^{2}}+U \frac{\partial U}{\partial x} \frac{\partial \ell}{\partial x}=0 .
$$

In terms of substantial derivative, Eq. (9) can be conveniently rewritten also as

$$
\frac{D^{2} \ell}{D t^{2}}-\frac{2}{\pi} \frac{1}{\varepsilon} \frac{\rho_{a}}{\rho_{l}} \frac{1}{H} \int_{0}^{1} \frac{\partial^{2} \ell}{\partial t^{2}} \ln |x-\xi| d \xi-\frac{U}{W e} \frac{\partial^{2} \ell}{\partial x^{2}}=0
$$


which is analogous to the classic one representing transverse vibrations of a finite length tensional string. Therefore, for $\rho_{a}=0$, Eq. (10) features two real and distinct characteristics on the entire physical domain, which are physically represented by traveling waves with velocities $\pm \sqrt{U / W e}$ relative to that of the basic flow $U$. Observe that the ambient pressure term expressed by the convolution integral models the so called added mass (to that of the oscillating liquid medium), whose effect is to vary the natural frequencies of the sheet with respect to the case of oscillation in a vacuum, i.e. $\rho_{a}=0$. As discussed in [15], Eq. 9 can be properly rearranged obtaining the following equivalent expression:

$$
\frac{\partial^{2} \ell}{\partial t^{2}}+U \frac{\partial^{2} \ell}{\partial t \partial x}+R U \int_{0}^{1} U \frac{\partial^{2} \ell}{\partial t \partial x} \ln |x-\xi| d \xi-\frac{1}{W e H} \frac{\partial^{2} \ell}{\partial x^{2}}=-U \frac{\partial v}{\partial x}+R U \int_{0}^{1} \frac{\partial v}{\partial t} \ln |x-\xi| d \xi
$$

where the parameter $R=2 \rho_{a} /\left(\pi \varepsilon \rho_{l}\right)$ has been introduced. According to Eq. (11), the oscillatory dynamics of the average sheet displacement $\ell$ appears forced by terms containing the lateral velocity $v$, which basically depends on the base flow $U$. The second and third terms at the left-hand side represent aerodynamic damping terms, since they contain the first time derivative of the displacement $\ell$. Note that the two damping coefficients, namely the basic flow velocity $U$ and the integral operator $R U \int_{0}^{1} U(\cdot) \ln |x-\xi| d \xi$, have opposite sign, and depending on the magnitude of the parameter $R$, the total damping coefficient can be negative. This makes the system unstable which, in the absence of nonlinear saturation, manifests an exponential amplification of disturbances [15].

\subsection{Linear stability analysis}

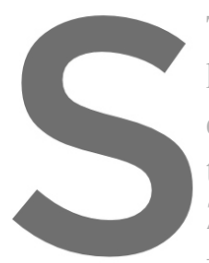

To analyze the asympto has been formulated. equations of the one-dir temporal modes positio $\lambda$ is the complex eigenvalue. This permits one to recas form
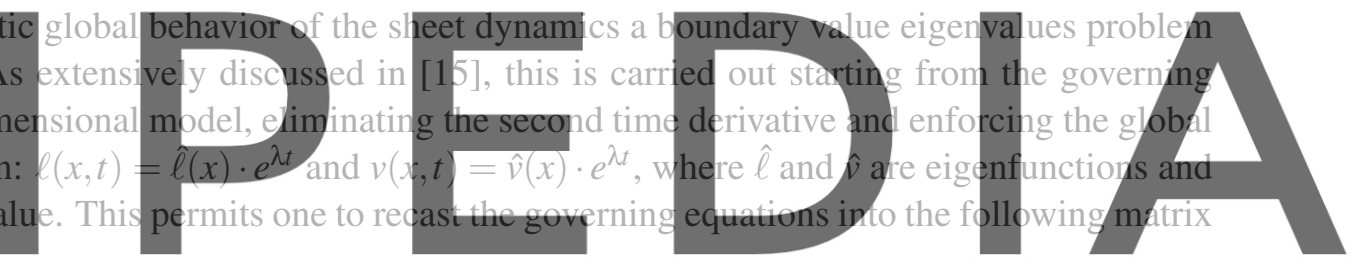

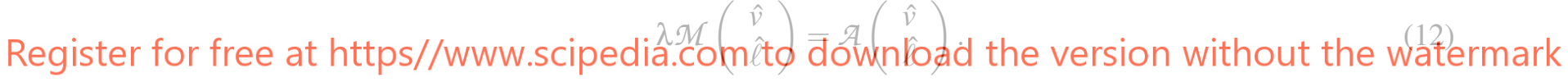

The reader is referred to [15] for a detailed explanation of the matrix operators $\mathcal{M}$ and $\mathcal{A}$ reported in Eq. 12. Eigenvalues and eigenfunctions are numerically computed by means of a Chebyshev collocation method in MATLAB environment, with both differential and integral terms being spectrally accurate.

\section{BASE CASE RESULTS}

The unsteady behavior of the liquid sheet forced by an impulse perturbation is reported in the present Section for the Base Case configuration (Table 1 of Section 2). Fig. 2 shows the snapshots of $v$ and $\ell$ perturbations obtained by numerical integration in MATLAB environment of the theoretical model, superimposed on VOF results. The initial $v$ perturbation splits in two down-winding traveling wave fronts which leave the domain through the right exit section at $t=0.22$ and $t=0.53$, respectively. Comparing these results with the expulsion times provided by the theoretical one-dimensional model [15], a good agreement is found, with differences of $2 \%$ and $7 \%$ for the fast and the slow traveling waves, respectively. The perturbation in $v$ produces a corresponding deformation of the meanline shape; this induced perturbation does not split in two wave fronts, as for the $v$ signal, but it exhibits a compact front 

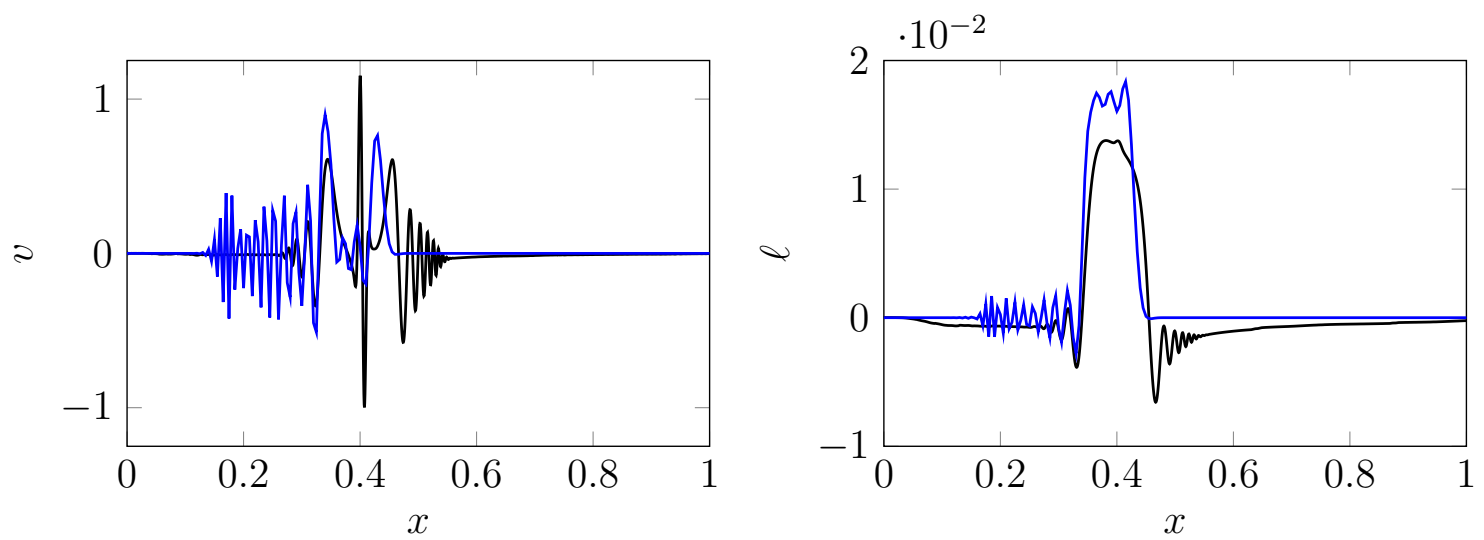

Figure 2: Instantaneous $v$ velocity component and meanline $\ell$ distributions at $t=0.05$ extracted from VOF simulation (black solid line) and computed from numerical integration of the one-dimensional model (blue solid line). $W e=2.5, \mathrm{Fr}=0.33, r_{\mathrm{p}}=0.01$.

whose ends travel downstream with the two characteristic speeds, which determines the enlargement of the perturbation front as $t$ increases. Results reported in Fig. 2 represent a validation of the VOF simulation, which is able to capture the underlying physics of the system under consideration. One of the main findings of the present investigation is that, after the complete expulsion of the perturbation from the computational domain, the meanline time evolution is characterized by low frequency sinuous

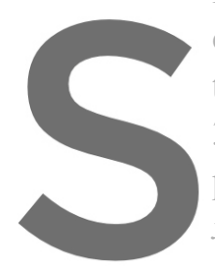
oscillations. This oscillat temporal signals stored 3, which reports frequenc presence of two low-frequency peaks is clearly $x=0.2$ the highest peak occ
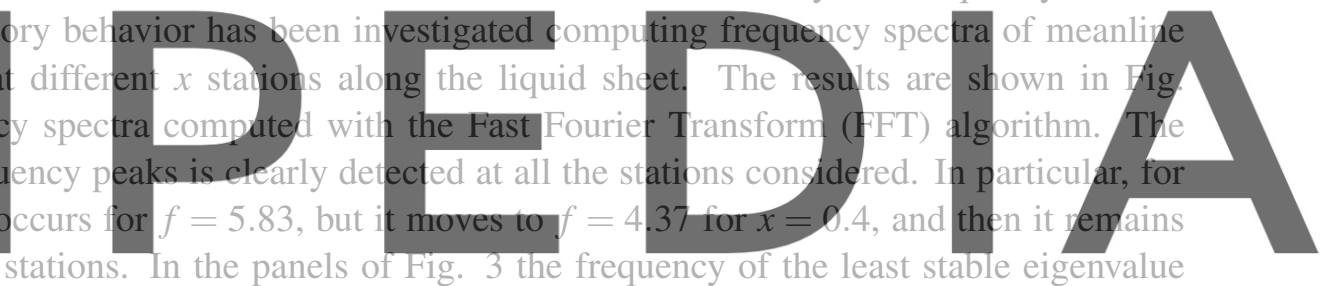

constant for all the other stations. In the panels of Fig. 3 the frequency of the least stable eigenvalue

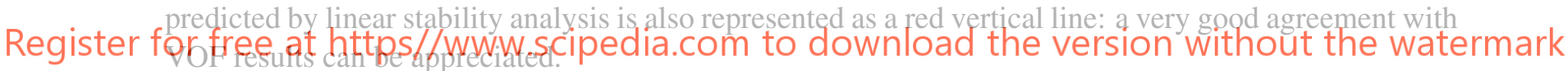

\section{PARAMETRIC ANALYSIS}

The effect of Weber number We and density ratio $r_{\rho}$ on the liquid sheet unsteady dynamics is examined in this Section. Two-dimensional flow fields obtained by means of VOF simulation are finally discussed for relevant conditions, providing comparisons with the work of Tammisola et al. [10], selected from the huge amount of literature papers for some interesting analogies.

\subsection{Influence of Weber number}

The variation of the Weber number $W e$ is initially performed by increasing the inlet mean velocity $U_{i}^{\star}=$ $0.70 \mathrm{~m} / \mathrm{s}$ and leaving the inlet sheet thickness unchanged, to not modify neither the slenderness parameter $\varepsilon$ nor the size of the computational domain $L^{\star}$. As a consequence, also the Froude number $F r$ varies, while clearly the density ratio $r_{\rho}$ is unaffected. The new set of dimensionless parameters considered for the VOF simulation is

- $W e=5, F r=0.66, \varepsilon=0.02, r_{\rho}=0.01$. 

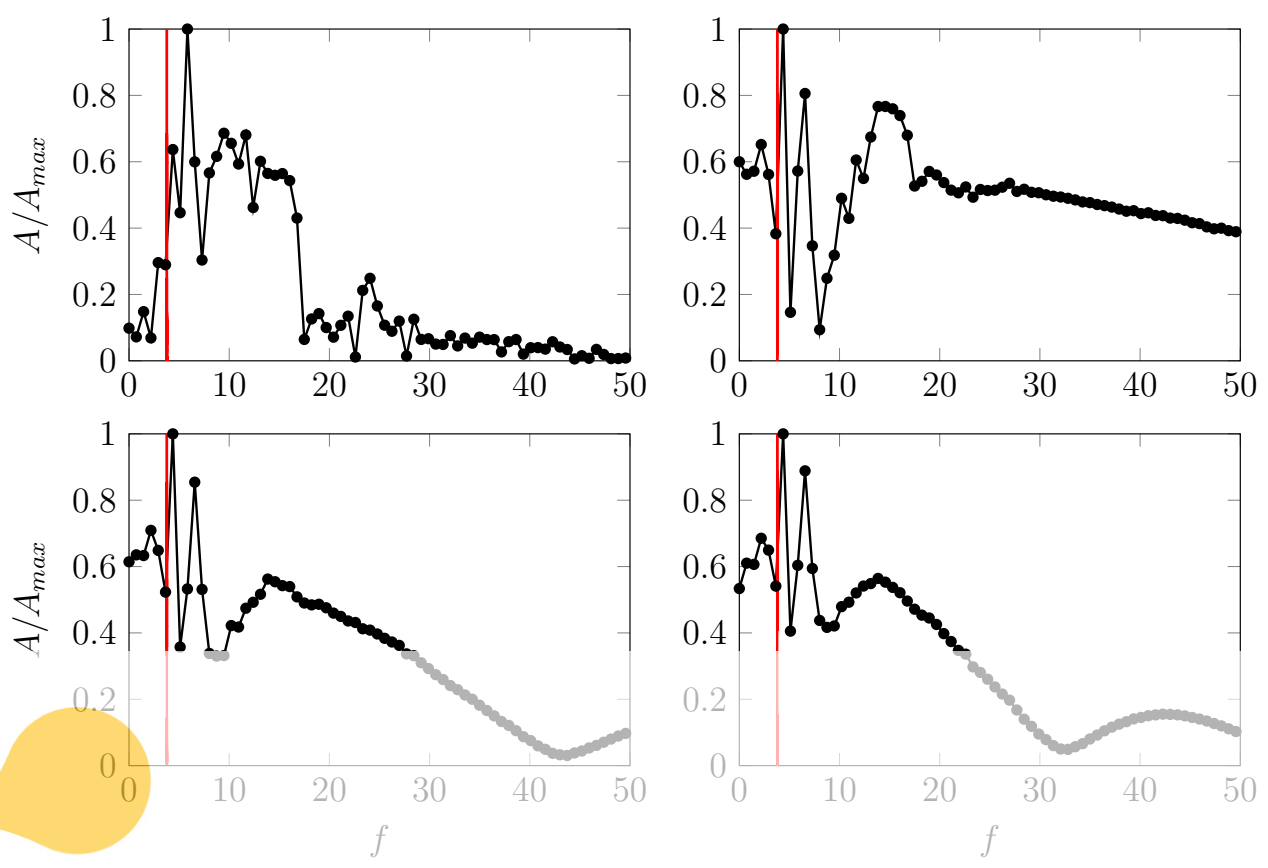

Figure 3: Fast Fourier Transform (FFT) of sheet meanline deflection extracted from VOF simulations at different $x$ stations. The red line represents the least stable frequency $\lambda_{i}=3.78$ arising from stability analysis. From left to right, top to bottom: $x=0.2,0.4,0.6,0.8 . W e=2.5, F r=0.33, r_{p}=0.01$

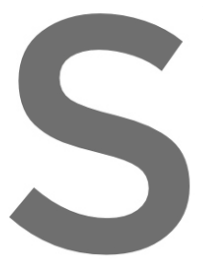
According to the prediction of the theoretical modet, crossing times with respect to the $B \varepsilon$ versa. This prediction is

4 , as regards the comparisc clearly recovered by the spa
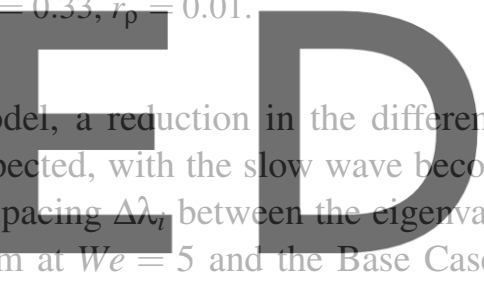

times arising from VOF simulation agree again well with the theoretical predictions, with differences of

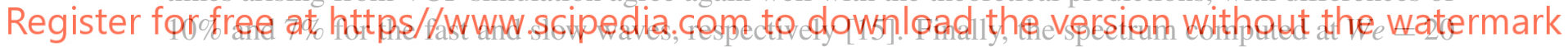

(where the surface tension is practically almost neglected) reveals the stabilizing effect of surface tension

on the gravitational liquid sheet, since the maximum growth rate increases with increasing We and the peak growth rate $\lambda_{r}$ moves towards higher frequencies $\lambda_{i}$ (see Fig. 4).

\subsection{Influence of density ratio}

The density ratio effect on the global unsteady dynamics of the liquid sheet is here investigated. Compared to the Base Case (Table 1), the gas density $\rho_{a}$ is the only parameter varied, and the following cases will be considered:

- $W e=2.5, F r=0.33, \varepsilon=0.02, r_{\rho}=0.001 ; 0.01 ; 0.05$,

where $r_{\rho}=0.01$ represents the Base Case extensively discussed in previous Section 4 . The density ratio effect on the system behavior predicted by the stability analysis is shown in Fig. 5, where the inner part of eigenvalues spectra is reported. While the case $r_{\rho}=0.001$ is interesting because it refers to a realistic air-water flow system, the case $r_{\rho}=0.05$ is analyzed because it represents the first value of density ratio for which flow instability is detected. It has to be stressed that in the case $r_{\rho}=0.05$ the 

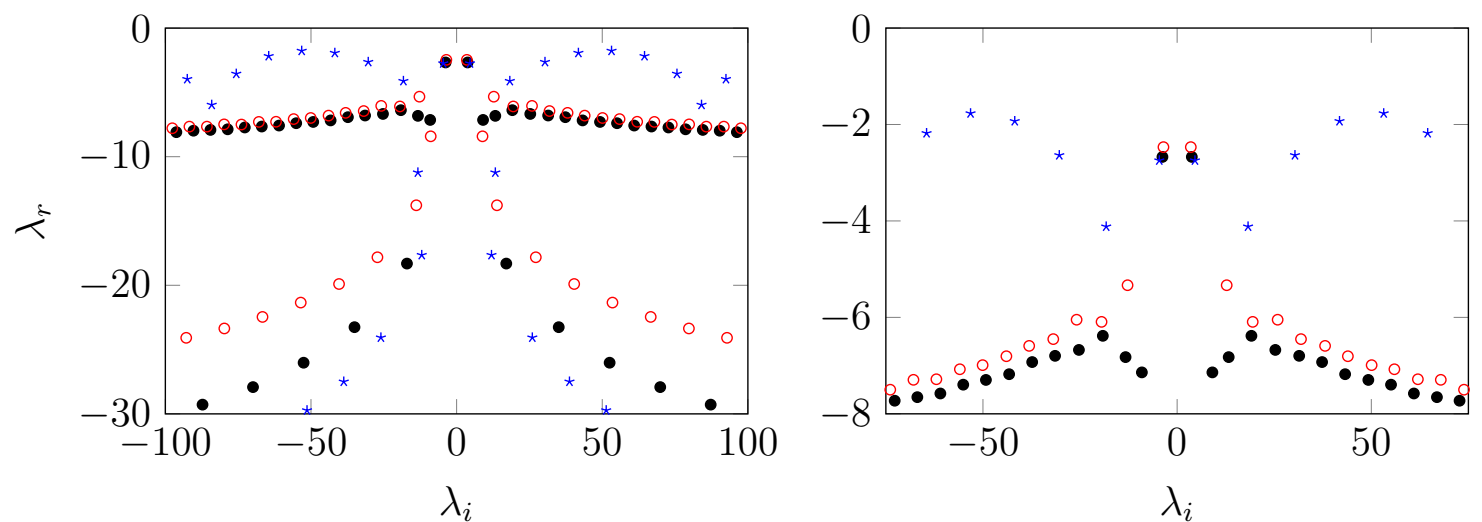

Figure 4: Weber number $W e$ effect at $r_{\rho}=0.01$ on the inner eigenvalues of spectrum (left), with a zoom on the upper branch around the least stable part (right). $W e=2.5$ (black filled dot); 5 (red unfilled dot); 20 (blue asterisk).

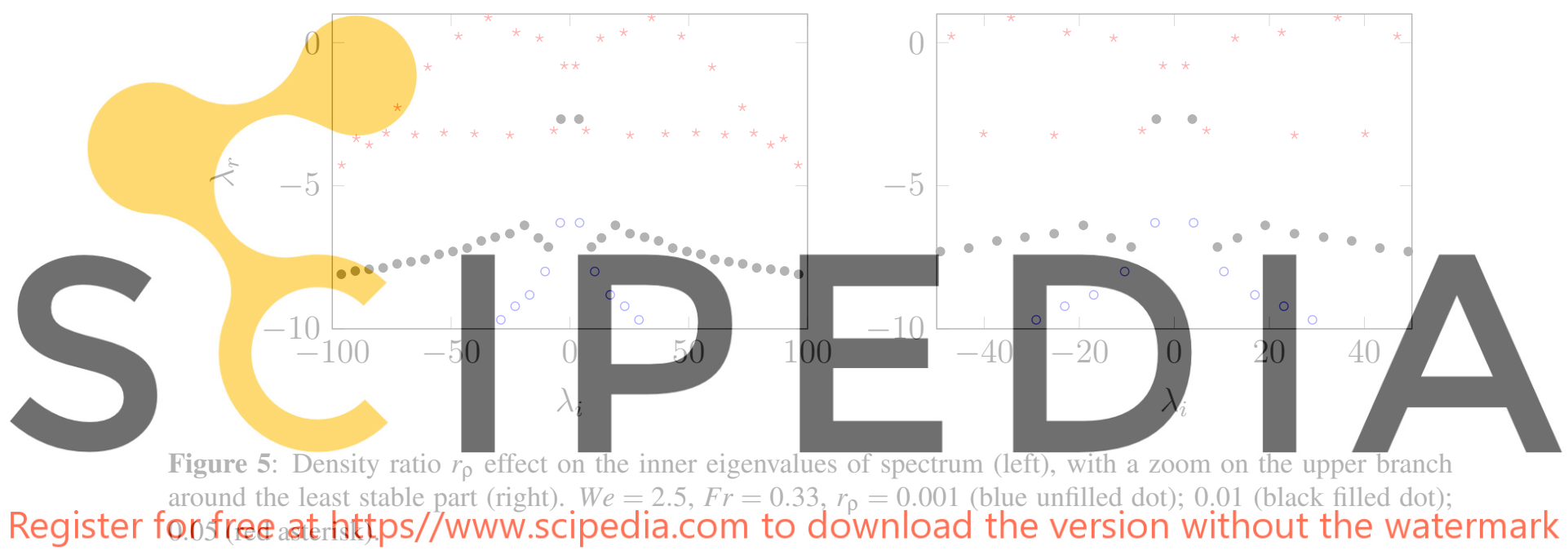

computational procedure described in Section 2 and aiready used for all the other cases presented in this work, has been reconsidered. In particular, the impulse perturbation represented by Eq. (5) has no longer been introduced; indeed, it has been noted that in case of unstable configuration, the rectangular initial condition itself represents a perturbation with respect to the theoretical steady base flow, which can eventually amplify and trigger the natural oscillations of the system.

\subsection{Two-dimensional patterns of unstable liquid sheet}

The occurrence of liquid sheet instability detected as the density ratio parameter $r_{\rho}$ increases makes the analysis of the whole two-dimensional flow field of great interest, to determine the presence of significant flow patterns associated with the unstable system behavior. With this objective in mind, a further VOF simulation of the following case has been performed:

- $W e=2.5, F r=0.33, r_{\rho}=0.1$,

for which the unstable behavior is much more evident than the case $r_{\rho}=0.05$ previously discussed. 


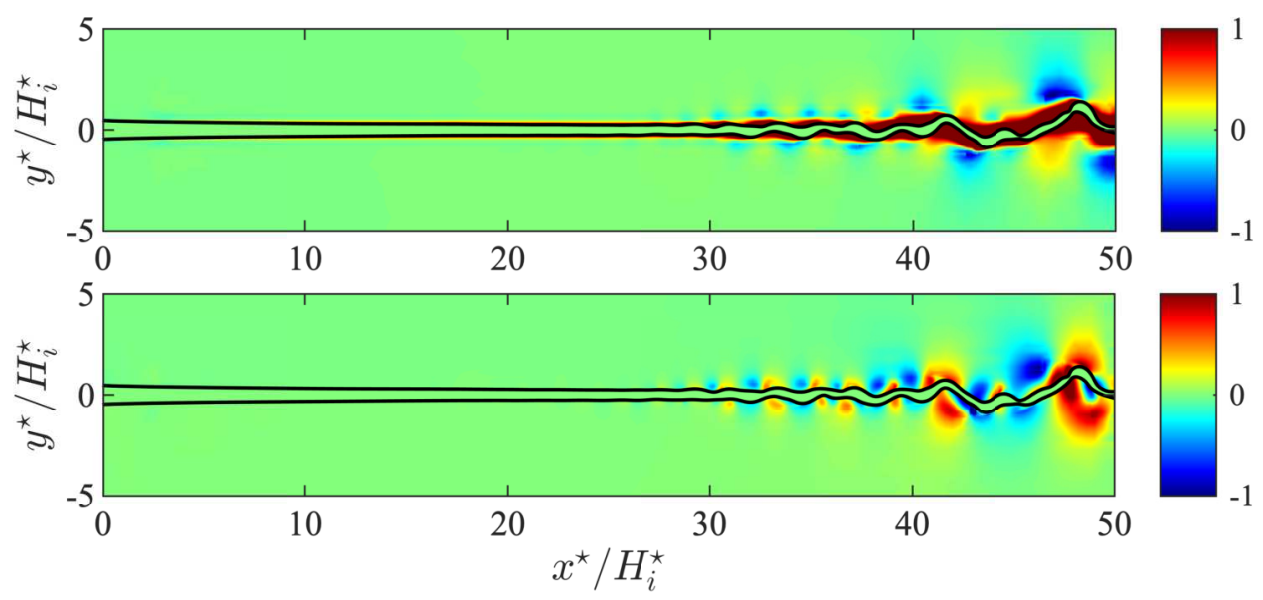

Figure 6: Instantaneous view of the ambient-phase velocity components $u_{a}^{\star} / U_{i}^{\star}$ (top) and $v^{\star} / U_{i}^{\star}$ (bottom) fields $W e=2.5, F r=0.33, r_{\rho}=0.1$. In all the panels the interface is highlighted by the black contour line.

Indeed, the unsteady oscillating dynamics of the flow for $r_{\rho}=0.1$ is clearly visible in Fig. 6, where an initial region of linear disturbance growth is followed by nonlinear saturation of the sheet pattern.

An analogy seems to exist between the global unstable behavior exhibited by the liquid sheet as the density ratio $r_{p}$ increases, and the surface tension-induced global instability found by Tammisola et al. flows of two immiscible fl instability for large enoug being the underlying phy two physical configuration tension is present) can be related to the density r
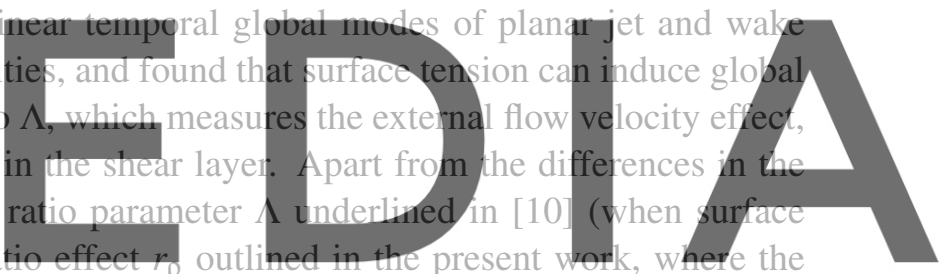

relatively strong entrainment effect of high density gas plays the destabilizing role. This analogy is also

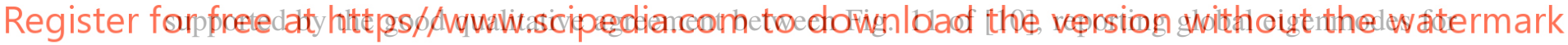
a wake flow $(\Lambda<1)$ for different values of the Weber number, and Fig. 6 of the present paper, showing the liquid sheet shape together with the distributions of velocity components $u_{a}$ and $v_{a}$ of the ambient gaseous phase for $r_{\rho}=0.1$. The oscillatory flow pattern exhibited by the $u$ eigenmode at $W e=5$ in Fig. 11(e) of [10] can be recovered in the $u_{a}$ distribution shown in Fig. 6, in particular for $30<x^{\star} / H_{i}^{\star}<40$, which is the liquid sheet region where the amplification of disturbances is not yet significantly affected by nonlinear effects. Furthermore, remember that in Section 3 it has been observed, Eq. (11), that the oscillatory dynamics of the average sheet displacement $\ell$ resembles that of a tensional string forced by terms containing the lateral velocity $v$, and subjected to a total damping coefficient which can assume negative values. Accordingly, one can argue that the distribution of velocity component $v_{a}$ of the ambient gaseous phase for $r_{\rho}=0.1$, showed in the bottom panel of Fig. 6 , represents such a forcing term which leads the system towards the instability when, for relatively high density ratios $r_{\rho}$, the total damping coefficient becomes negative. The comparison with wake rather than jet flows of Tammisola et al. [10] is motivated by the observation that the inner stream acceleration and interface contraction experienced by wake flows (Fig. 1 of [10]), due to the shear effect of the higher velocity outer stream, can be regarded as the counterpart of the gravity effect considered in the present work. On the other hand, the analogy seems 
to fail in the initial part of the sheet where the shear induced velocity of the gaseous phase is weaker. Finally, it has also to be stressed that the surface tension plays a destabilizing role on the flow fields studied in [10], whilst for the gravitational liquid sheet which is the object of the present investigation, the surface tension is stabilizing, as already found by Girfoglio et al. [8] for the nappe problem.

\section{CONCLUSIONS}

The unsteady dynamics of planar liquid sheet flows freely falling in the gravitational field in supercritical regimes and surrounded on both sides by quiescent gaseous environments has been numerically investigated by means of the Volume-Of-Fluid (VOF) technique. Surface tension at the interface of the two phases and viscosity effects have been included in the computations, carried out by means of the open source BASILISK code. It takes advantage of an adaptive-grid strategy, which has been exploited to retain the maximum level of refinement at the gas-liquid interface, adapting dynamically the grid to its spatiotemporal evolution. The global behavior of the non-parallel liquid flow has been analyzed by perturbing the initial steady configuration by means of a Gaussian bump in the transverse velocity component of relatively small amplitude, thereby guaranteeing the linearity of the system response. To validate the numerical results, and to gain more physical insights on the fluid system, a linear one-dimensional model has been developed. A physical interpretation of the theoretical model relates the sheet sinuous dynamics to transverse vibrations of a tensional string forced by terms containing the lateral velocity, and subjected to a total damping which can assume negative values. It features two real and distinct characteristics on the entire physical domain, which are physically represented by traveling waves with velocities $U \pm \sqrt{U / W e}$. Global 1D sinuous eigenmodes and eigenvalues have been evaluated by using the normal modes approach. To effectively compare VOF simulations data to findings of the linear stability theory, a suited one-dimensional reduction technique applied to the VOF fields has been implemented. Such a reduction technique has allowed one not only to compare the average sheet displacements (eigenmodes) obtained with both the procedures, but also to extract from the VOF data the peak frequencies of the sheet centreline oscillations via FFT analysis.

The VOF computation of the average sheet shape and natural oscillation frequency closely agrees with predictions of the one-dimensional theoretical model. A parametric investigation has been also performed, to analyze in particular the influence of Weber number and density ratio. The results showed that the surface tension plays a stabilizing role. The major novel finding is that the sheet becomes unstable for relatively high value of density ratio, thus confirming the theoretical prediction. Since the oscillatory dynamics of the average sheet displacement resembles that of a tensional string forced by terms containing the lateral velocity, and subjected to a total damping coefficient which can assume negative values, it has been argued that the distribution of transverse velocity component of the ambient gaseous phase represents such a forcing term which leads the system towards the instability when, for relatively high density ratios, the total damping becomes negative. The VOF simulations have also highlighted the convective character of the instability. Therefore, an analogy seems to exist between the global unstable behavior exhibited by the liquid sheet as the density ratio increases, and the shear-induced instability due to the wave motions of both flow streams on the interface, when the surface tension is present, found by Tammisola et al. [10]. This analogy is supported by the qualitative agreement between global eigenmodes for the wake flow shown in [10], and present distributions of velocity components of the ambient gaseous phase for $r_{\rho}=0.1$. However, while the surface tension plays a destabilizing role on the flow fields studied in [10], for the gravitational liquid sheet the surface tension is stabilizing. 


\section{REFERENCES}

[1] H. B. Squire, Investigation of the instability of a moving liquid film, Br. J. Appl. Phys. 4, 167-169 (1953).

[2] S. J. Weinstein, D. S. Ross, J. R. Kenneth and N. S. Barlow, On oblique liquid curtains, J. Fluid Mech. 876, 1-9 (2019).

[3] D. J. Duke, D. Honnery and J. Soria, The growth of instabilities in annular liquid sheets, Exp. Therm. Fluid Sci. 68, 89-99 (2015).

[4] A. Zandian, W. A. Sirignano and F. Hussain, Planar liquid jet: early deformation and atomization cascades, Phys. Fluids 29, 062109 (2017).

[5] H. Lhuissier, P. Brunet and S. Dorbolo, Blowing a liquid curtain, J. Fluid Mech. 795, 784-807 (2016).

[6] L. de Luca and M. Costa, Instability of a spatially developing liquid sheet, J. Fluid Mech. 331, 127-144 (1997).

[7] O. Tammisola, A. Sasaki, F. Lundell, M. Matsubara and L. D. Soderberg, Stabilizing effect of surrounding gas flow on a plane liquid sheet, J. Fluid Mech. 672, 5-32 (2011).

[8] M. Girfoglio, F. De Rosa, G. Coppola and L. de Luca, Unsteady critical liquid sheet flows, J. Fluid Mech. 821, 219-247 (2017).

[9] S. Schmidt and K. Oberleithner, Instability of forced planar liquid jets: mean field analysis and non linear simulation, J. Fluid Mech. 883, 1-38 (2020).

[10] O. Tammisola, F. Lundell and L. D. Soderberg, Surface tension-induced global instability of planar jets and wakes, J. Fluid Mech. 713, 632-658 (2012).

[11] R. Scardovelli and S. Zaleski, Direct numerical simulation of free-surface and interfacial flow, Annu. Rev. Fluid Mech. 31, 567-603 (1999).

[12] S. Popinet, Gerris: a tree-based adaptive solver for the incompressible Euler equations in complex geometries, J. Comput.Phys. 190, 572-600 (2003).

[13] S. Popinet, An accurate adaptive solver for surface-tension-driven interfacial flows, J. Comput.Phys. 228, 5838-5866 (2009).

[14] J. A. van Hooft, S. Popinet, C. C. van Heerwaarden, S. J. A. van der Linden, S. R. de Roode and B. J. H. van de Wiel, Towards adaptive grids for atmospheric boundary- layer simulations, BoundaryLayer Meteorol. 167, 421-443 (2018).

[15] A. Della Pia, M. Chiatto and L. de Luca, Global eigenmodes of thin liquid sheets by means of Volume-of-Fluid simulations, Phys. Fluids 32, 082112 (2020). 